\title{
Relationship between homocysteine levels and post-stroke cognitive impairment in female and male population: from a prospective multicenter study
}

\begin{abstract}
Runzhi Lii, ${ }^{1,2}$ Haoyi Weng, ${ }^{3,8,9}$, Yuesong Pan ${ }^{1,4}$, Xia Meng', ${ }^{1,4}$ Xiaoling Liao ${ }^{1,4}$, Mengxing Wang ${ }^{1,4}$, Yuan Zhang ${ }^{1,4}$, Yi Sui ${ }^{5}$, Lijun Zuo ${ }^{1,4}$, Yanli Wang ${ }^{1,4}$, Ziyan Jia ${ }^{1,4}$, Mengfan Sun ${ }^{1,4}$, Wenyi $\mathrm{Li}^{1,4}$, Yaou Liu ${ }^{2}$, Jinglong $\mathrm{Chen}^{6}$, Jun $\mathrm{Xu}^{4,7 *}$, Yongjun Wang ${ }^{1,4 *}$

'Department of Neurology, Beijing Tiantan Hospital, Capital Medical University, Beijing 100050, China;

${ }^{2}$ Department of Radiology, Beijing Tiantan Hospital, Capital Medical University, Beijing 100050, China; ${ }^{3}$ Bioinformatics Department, Shenzhen WeGene Clinical Laboratory, Shenzhen 518118, Guangdong Province, China; ${ }^{4}$ China National Clinical Research Center for Neurological Diseases, Beijing 100070, China;

${ }^{5}$ Shenyang First People's Hospital, Shenyang Medical College Affiliated Shenyang Brain Hospital, Shenyang 110041, Liaoning Province, China;

${ }^{6}$ Department of Geriatric Medicine, China National Clinical Key Specialty, Guangzhou First People's Hospital; School of Medicine, South China University of Technology, Guangzhou 510180, Guangdong Province, China; 'Department of Cognitive Neurology, Beijing Tiantan Hospital, Capital Medical University, Beijing 100070, China;

${ }^{8}$ WeGene, Shenzhen Zaozhidao Technology Co. Ltd, Shenzhen 518118, Guangdong Province, China; ${ }^{9}$ Hunan Provincial Key Lab on Bioinformatics, School of Science and Engineering, Central South University, Changsha 410083, Hunan Province, China
\end{abstract}

'These authors contributed equally as corresponding authors.

Address for Correspondence: Yongjun Wang and Jun Xu,

No. 119, South 4th Ring West Road,

Fengtai District, Beijing 100070, China E-mail: yongjunwang@ncrcnd.org.cn (Wang Y.); neurojun@126.com (Xu J.)

\begin{tabular}{|l|}
\hline Access this article online \\
Website: \\
www.intern-med.com \\
\hline DOI: \\
10.2478/jtim-2021-0035 \\
\hline Quick Response Code: \\
\hline \\
\hline
\end{tabular}

\section{ABSTRACT}

Background and Objectives: To investigate the relationship between homocysteine levels and post-stroke cognitive impairment (PSCl) in Chinese female and male populations with minor acute ischemic stroke or transient ischemic attack. Materials and methods: A total of 1070 participants with clinically confirmed acute minor ischemic stroke or transient ischemic attack and baseline homocysteine information from a nationwide multicenter prospective registry study in China were included in this study. Of these, 919 patients had cognitive assessments at 3-month follow-ups and 584 participants had cognitive assessments at 12-month follow-ups. The incidence of PSCl was defined as a Montreal Cognitive Assessment score $\leq 22$. The differences in homocysteine levels and the incidence of PSCl were compared between female and male populations. Relationships between homocysteine levels and the incidence of $\mathrm{PSCl}$ in female and male populations were analyzed using multiple logistic regression, respectively. Results: Females had lower baseline homocysteine levels than males. Compared to males, females had lower education levels, lower rates of smoking and alcohol intake, and higher rates of diabetes and hypertension. No relationship was observed between elevated homocysteine level and 3-month PSCl incidence in either females or males. After adjusting the confounders, elevated baseline homocysteine significantly increased the 12-month PSCl risk (odds ratio $3.28,95 \%$ confidence interval $1.47-7.34, P=0.004$ ) in females, but not in males (odds ratio $0.86,95 \%$ confidence interval $0.49-1.49, P=0.586$ ). Conclusion: Elevated homocysteine levels increased the 12-month PSCl risk in females, but not in males with minor acute ischemic stroke or transient ischemic attack.

Key words: homocysteine, stroke, cognitive impairment, sex difference, female

\section{INTRODUCTION}

Minor stroke and transient ischemic attacks (TIAs) are the common manifestations of acute cerebrovascular events. ${ }^{[1]}$ Poststroke cognitive impairment (PSCI) is one of the major residual impairments and is associated with functional outcomes and 
survival in minor stroke and TIA patients. ${ }^{[2,3]}$ Homocysteine is an important intermediate in methionine, folate, and onecarbon metabolism, and elevated homocysteine increases the risk of stroke and age-associated cognitive impairment. ${ }^{[4]}$ Homocysteine may be a prognostic biomarker or a modifiable target for preventing PSCI. ${ }^{[8]}$

Previous studies that demonstrated the relationship between homocysteine and PSCI have yielded inconsistent results. ${ }^{[8-12]}$ A cohort study $(n=81)^{[11]}$ demonstrated that homocysteine was associated with vascular dementia at 3 months after stroke. A case-control study (82 cases vs. 80 controls $)^{[10]}$ demonstrated that homocysteine levels were correlated with cognitive impairment after stroke at 3-month follow-up. ${ }^{[10]}$ However, a larger sample size case-control study (169 cases vs. 103 controls ${ }^{[12]}$ demonstrated opposite results that there were no associations between homocysteine and PSCI after 3-6 months. A prospective cohort study $(n=251)$ demonstrated that homocysteine levels at 3 months had no relationship with vascular dementia at 3-month follow-ups after stroke. ${ }^{[9]}$ In summary, the evidence on the relationship between homocysteine levels and PSCI is limited. Also, (1) previous studies had small sample size and inconsistent conclusions in the relationship between homocysteine and PSCI and (2) cognitive follow-ups in previous studies exploring the relationship between homocysteine levels and PSCI were for short term (3-6 months). Additionally, homocysteine concentration was associated with sex. ${ }^{[13,14]}$ Females had lower homocysteine levels than males. ${ }^{[15-18]}$ But females were more susceptible to the damaging effect of homocysteine in vascular diseases, such as the risk of ischemic stroke and poor prognosis (death and major disability) of acute ischemic stroke, than males. ${ }^{[15,16,18]}$ But the correlation of homocysteine and PSCI has not been elucidated in female and male populations.

Therefore, in this study, we aimed to explore the association between the homocysteine levels and the risk of short- and long-term PSCI in male and female patients with minor acute ischemic stroke or TIA in a prospective multicenter cohort study.

\section{MATERIALS AND METHODS}

\section{Study design}

The Impairment of Cognition and Sleep after acute ischemic stroke or transient ischemic attack in Chinese patients (ICONS) study is a nationwide multicenter, prospective registry study investigating the occurrence and associated factors of cognitive impairment and sleep disorder after acute ischemic stroke or TIA. ${ }^{[19]}$ The rationale and design of the ICONS have been described in detail elsewhere. ${ }^{[19]}$ The ICONS study enrolled 2625 subjects and excluded prior diagnosis of cognitive impairment. The study was performed according to the principles expressed in the Declaration of Helsinki. The protocol of the ICONS study was approved by the ethics committee of a hospital (blinded as requested; Institutional Review Board approval number KY2015-001-01) and all participating centers. All participants obtained written informed consent from patients or legally authorized representatives.

In the current study, patients who had baseline homocysteine levels and cognitive assessment at 3 or 12 months were included. A total of 1070 participants with clinically confirmed acute minor ischemic stroke or TIA and baseline homocysteine information were included in this study, of which 919 patients had 3-month cognitive assessments and 584 participants had 12-month cognitive assessments. Figure 1 shows the flowchart of participant enrollment.

\section{Clinical variables}

Patient demographics, medical histories, risk factors of vascular disease, and the National Institutes of Health Stroke Scale (NIHSS) scores at baseline were collected by an electronic data capture system from an electronic record system. Cognitive function was evaluated using the Beijing version of Montreal Cognitive Assessment (MoCA). ${ }^{[17]}$ MoCA evaluations at 3 and 12 months were conducted face to face by trained examiners. The incidences of PSCI at 3- and 12-month follow-up were defined as MoCA scores $\leq 22 .{ }^{[17]}$ The definition of minor acute ischemic stroke was according to NIHSS $\leq 3 .{ }^{[20]}$ Elevated homocysteine was defined as $\geq 15 \mu \mathrm{mol} / \mathrm{L}^{.{ }^{[21]}}$ In the analysis, the baseline homocysteine levels were dichotomized according to 15 $\mu \mathrm{mol} / \mathrm{L}$ or categorized according to tertiles. ${ }^{[2]}$

\section{Blood biochemical index}

Fasting blood samples were collected in serum separation tubes and ethylenediaminetetraacetic acid anticoagulation blood collection tubes on the second day of hospitalization. Plasma levels of homocysteine, serum folate, vitamin B12, and creatinine were measured using automated analyzers at the core laboratory of a hospital (blinded as requested). Plasma total homocysteine was measured using the enzyme rate method (tailed). Serum folate was measured using a chemiluminescent immunoassay (Beckman Coulter; Beckman Coulter, Inc, CA, USA). Serum vitamin B12 was measured using an automated chemiluminescence system (Siemens ADVIA Centaur XP, NY, USA). The estimated glomerular filtration rate (eGFR) was calculated using the Chronic Kidney Disease Epidemiology Collaboration equation.

\section{Statistical analysis}

Continuous variable was presented as median with interquartile range or mean \pm standard deviation. Categorical variable was presented as percentage. Baseline characteristics were analyzed by the $\chi^{2}$ test or the Fisher's 


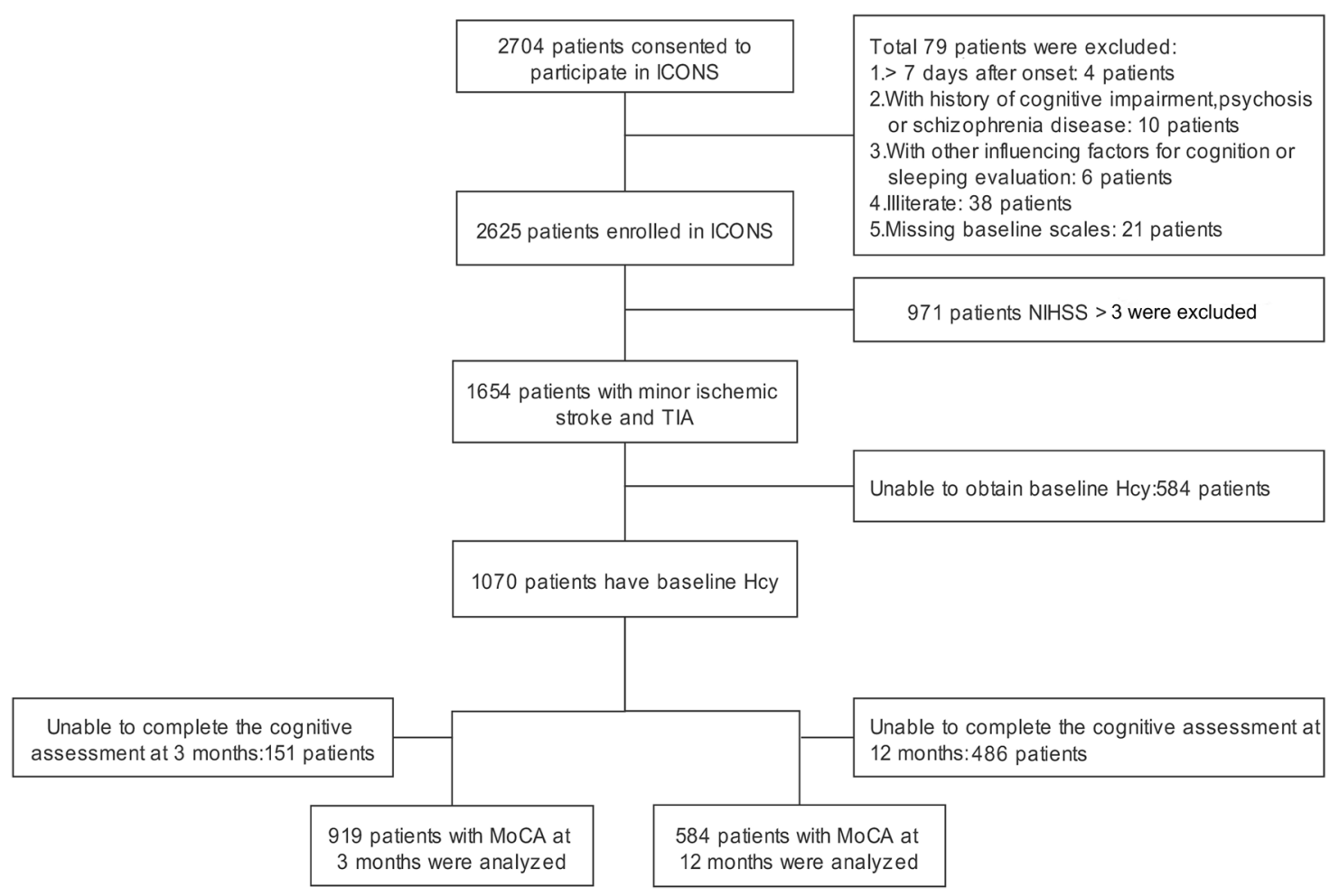

Figure 1. Flowchart of participant enrollment. Hcy: homocysteine; ICONS: The Impairment of Cognition and Sleep after acute ischemic stroke or transient ischemic attack in Chinese patients; MoCA: Montreal Cognitive Assessment; NIHSS: National Institutes of Health Stroke Scale; TIA: transient ischemic attack.

exact test for the categorical variables and by the $t$-test or the Mann-Whitney test for the continuous variables. The differences in homocysteine levels and the incidence of PSCI were compared between female and male populations using the $t$-test and $\chi^{2}$ test, respectively. Confounding factors included age, education, NIHSS, diabetes mellitus, current smoking, baseline levels of folate, vitamin B12, eGFR, and methylenetetrahydrofolate reductase (MTHFR) genotype. Imputation of the missing values of the covariates studied was performed with the method of chained equations, generating five imputed data sets. Multiple logistic regression analysis for the association of homocysteine and PSCI was performed on each imputed data set, and results including odds ratios (ORs) and 95\% confidence intervals (CIs) were derived by combining the output of the five imputed multiple analyses. Variables with imputed values and the respective numbers are listed in detain in Table S1. A two-sided $P<0.05$ was considered to indicate statistical significance. All analyses were conducted with SAS software, version 9.4 (SAS Institute Inc, NC, USA).

\section{RESULTS}

\section{Baseline demographics and clinical characteristics}

The baseline demographic and clinical characteristics of patients with 3 months of cognitive follow-up are shown in
Table 1. In the 919 patients with 3-month cognitive followup, compared to males, females had lower homocysteine levels $(14.1 v s .18 .1 \mu \mathrm{mol} / \mathrm{L} ; P<0.001)$. Females were older and had lower education level, higher rates of diabetes mellitus, hypertension, and coronary heart disease, lower levels of folate, vitamin B12, and eGFR, and a lower rate of smoking and alcohol intake. Compared to females with homocysteine $<15 \mu \mathrm{mol} / \mathrm{L}$, females with elevated homocysteine levels $(\geq 15 \mu \mathrm{mol} / \mathrm{L})$ were older and had a higher rate of stroke history and lower levels of vitamin $\mathrm{B}_{12}$ and eGFR. Compared to males with homocysteine $<15 \mu \mathrm{mol} / \mathrm{L}$, those with elevated homocysteine levels were older and had lower rate of diabetes mellitus, lower levels of vitamin B12 and eGFR, and higher creatinine levels.

Compared to the included patients $(n=584)$ with 12 -month MoCA, the excluded patients $(n=491)$ had balance characteristics in age, body mass index (BMI), education, MTHFR C667T genotype, and kidney function including creatinine and eGFR, but had lower NIHSS, lower homocysteine, and higher folate and vitamin B12 levels (Table S2). The baseline demographic and clinical characteristics of patients with 12-month cognitive followup are shown in Table 2 . In the 584 patients with 12-month cognitive follow-up, compared to males, females had lower homocysteine (14.2 vs. $18.4 \mu \mathrm{mol} / \mathrm{L} ; P<0.001)$. Females had lower education, higher rates of diabetes mellitus and 
Li et al.: Homocysteine levels and post-stroke cognitive impairment

\begin{tabular}{|c|c|c|c|c|c|c|}
\hline \multirow[t]{2}{*}{ Baseline characteristics } & \multicolumn{2}{|l|}{ Overall } & \multicolumn{2}{|l|}{ Female } & \multicolumn{2}{|l|}{ Male } \\
\hline & $\begin{array}{l}\text { Female } \\
(n=667)\end{array}$ & $\begin{array}{l}\text { Male } \\
(n=252)\end{array}$ & $\begin{array}{l}\text { Hcy } \geq 15 \mu \mathrm{mol} / \mathrm{L} \\
(n=111)\end{array}$ & $\begin{array}{l}\text { Hcy }<15 \\
\mu \mathrm{mol} / \mathrm{L} \\
(n=141)\end{array}$ & $\begin{array}{l}\text { Hcy } \geq 15 \mu \mathrm{mol} / \mathrm{L} \\
(n=459)\end{array}$ & $\begin{array}{l}\text { Hcy }<15 \\
\mu \mathrm{mol} / \mathrm{L} \\
(n=208)\end{array}$ \\
\hline Age (years), mean $\pm S D$ & $63.25 \pm 10.54$ & $\begin{array}{l}60.45 \pm \\
11.17^{*}\end{array}$ & $64.9 \pm 11.6$ & $61.9 \pm 9.5^{*}$ & $61.2 \pm 11.6$ & $58.9 \pm 10.1^{*}$ \\
\hline Education $\leq 9$ years $(\%)$ & $190(75.40)$ & $399(59.82)^{*}$ & $86(77.5)$ & $104(73.8)$ & $276(60.1)$ & $123(59.1)$ \\
\hline $\mathrm{BMI}\left(\mathrm{kg} / \mathrm{m}^{2}\right)$, mean $\pm \mathrm{SD}$ & $25.40 \pm 3.61$ & $24.90 \pm 3.15$ & $25.5 \pm 3.8$ & $25.3 \pm 3.5$ & $24.9 \pm 3.1$ & $25 \pm 3.4$ \\
\hline NIHSS, mean \pm SD & $1.49 \pm 1.31$ & $1.46 \pm 1.19$ & $1.4 \pm 1.2$ & $1.5 \pm 1.4$ & $1.4 \pm 1.1$ & $1.6 \pm 1.3$ \\
\hline \multicolumn{7}{|l|}{ Medical history } \\
\hline Diabetes mellitus (\%) & $74(29.37)$ & $127(19.04)^{*}$ & $30(27.0)$ & $44(31.2)$ & $66(14.4)$ & $61(29.3)^{*}$ \\
\hline Hypertension (\%) & $181(71.83)$ & $397(59.52)^{*}$ & $85(76.6)$ & $96(68.1)$ & $276(60.1)$ & $121(58.2)$ \\
\hline Hyperlipidemia (\%) & $28(11.11)$ & 76 (11.39) & $10(9)$ & $18(12.8)$ & $46(10)$ & $30(14.4)$ \\
\hline History of stroke (\%) & $54(21.43)$ & $143(21.44)$ & $28(25.2)$ & $26(18.4)$ & $102(22.2)$ & $41(19.7)$ \\
\hline History of TIA (\%) & $15(5.95)$ & $35(5.25)$ & $5(4.5)$ & $10(7.1)$ & $25(5.5)$ & $10(4.8)$ \\
\hline History of $\mathrm{CHD}(\%)$ & $49(19.44)$ & $60(9.00)^{*}$ & $19(17.1)$ & $30(21.3)$ & $44(9.6)$ & $16(7.7)$ \\
\hline Current smoking (\%) & $8(3.17)$ & $326(48.88)^{*}$ & $6(5.4)$ & $2(1.4)$ & $227(49.5)$ & $99(47.6)$ \\
\hline Alcohol intake (\%) & $2(0.79)$ & $163(24.44)^{*}$ & $1(0.9)$ & $1(0.7)$ & $108(23.5)$ & $55(26.4)$ \\
\hline \multicolumn{7}{|l|}{ Laboratory results } \\
\hline $\begin{array}{l}\text { Folate (nmol/L), median } \\
\text { (IQR) }\end{array}$ & $\begin{array}{l}10.59(5.5- \\
17.3)\end{array}$ & $\begin{array}{l}8.11(4.2- \\
14.4)^{*}\end{array}$ & $9.7(5.9-15.5)$ & $\begin{array}{l}12.0(5.3- \\
18.4)\end{array}$ & $7.1(4.0-2.9)$ & $\begin{array}{l}10.8(5.2- \\
16.9)^{*}\end{array}$ \\
\hline $\begin{array}{l}\text { Vitamin B12 (pmol/L), } \\
\text { median (IOR) }\end{array}$ & $\begin{array}{l}295.5(188.5- \\
441.0)\end{array}$ & $\begin{array}{l}236(170.0- \\
354.0)^{*}\end{array}$ & $\begin{array}{l}241.5(162.5- \\
378.0)\end{array}$ & $\begin{array}{l}339.5(232.0- \\
506.0)^{*}\end{array}$ & $\begin{array}{l}217.0(158.0- \\
314.0)\end{array}$ & $\begin{array}{l}297.0(216.5- \\
494.0)^{*}\end{array}$ \\
\hline $\operatorname{Hcy}(\mu \mathrm{mol} / \mathrm{L})$, median (IOR) & $\begin{array}{l}14.1(11.5- \\
17.8)\end{array}$ & $\begin{array}{l}18.1(14- \\
24.5)^{*}\end{array}$ & $\begin{array}{l}18.1(16.4- \\
23.3)\end{array}$ & $\begin{array}{l}11.8(10.2- \\
13.3)^{*}\end{array}$ & $\begin{array}{l}21.1(17.7- \\
29.5)\end{array}$ & $\begin{array}{l}12.7(11.3- \\
13.8)^{*}\end{array}$ \\
\hline $\begin{array}{l}\text { Creatinine }(\mu \mathrm{mol} / \mathrm{L}) \text {, median } \\
\text { (IQR) }\end{array}$ & $56(49-66)$ & $73(66-82)^{*}$ & $59(51-70)$ & $54(48-62)^{*}$ & $75(67-84)$ & $71(63-78)^{*}$ \\
\hline $\begin{array}{l}\text { eGFR }\left(\mathrm{mL} / \mathrm{min} / 1.73 \mathrm{~m}^{2}\right) \text {, } \\
\text { median (IQR) }\end{array}$ & $\begin{array}{l}93.51(83.2- \\
103.2)\end{array}$ & $\begin{array}{l}94.61(85.1- \\
102.4)\end{array}$ & $\begin{array}{l}88.6(77.7- \\
99.6)\end{array}$ & $\begin{array}{l}96.6(87.8- \\
104.2)^{*}\end{array}$ & $\begin{array}{l}92.8(82.6- \\
101.1)\end{array}$ & $\begin{array}{l}97.7(90.9- \\
105.7)^{*}\end{array}$ \\
\hline \multicolumn{7}{|l|}{ MTHFR C667T } \\
\hline $\mathrm{CC}(\%)$ & 49 (19.68) & $141(22.1)$ & $18(16.7)$ & $31(22.0)$ & $93(26.9)$ & $48(24.9)$ \\
\hline СТ (\%) & $112(44.98)$ & $296(46.39)$ & $43(39.8)$ & 69 (48.9) & $184(41.3)$ & $112(58.0)$ \\
\hline TT (\%) & $88(35.34)$ & 201 (31.05) & $47(43.5)$ & $41(29.1)$ & $168(37.8)$ & $33(17.1)$ \\
\hline $\begin{array}{l}\text { MoCA at } 3 \text { months (mean } \\
\pm \text { SD) }\end{array}$ & $23.77 \pm 5.56$ & $25.06 \pm 4.27^{*}$ & $23.41 \pm 6.13$ & $24.05 \pm 5.07$ & $24.97 \pm 4.35$ & $25.27 \pm 4.1$ \\
\hline 3-month PSCI (\%) & $81(32.14)$ & $150(22.49)^{*}$ & $39(35.14)$ & $42(29.79)$ & $106(23.09)$ & $44(21.15)$ \\
\hline
\end{tabular}

${ }^{*}$ Significant difference between two groups, $P<0.05$. BMI: body mass index; CHD: coronary heart disease; eGFR: estimated glomerular filtration rate; Hcy: homocysteine; IQR: interquartile range; MoCA: Montreal Cognitive Assessment; MTHFR: methylenetetrahydrofolate reductase; NIHSS: National Institutes of Health Stroke Scale; PSCI: post-stroke cognitive impairment; SD: standard deviation; TIA: transient ischemic attack.

hypertension, higher levels of folate and vitamin B12, and a lower rate of smoking and alcohol intake. Compared to females with homocysteine $<15 \mu \mathrm{mol} / \mathrm{L}$, females with elevated homocysteine levels were older, had a higher rate of stroke history, and lower levels of vitamin B12 and eGFR. Compared to males with homocysteine $<15$ $\mu \mathrm{mol} / \mathrm{L}$, those with elevated homocysteine levels were older, had a lower rate of diabetes mellitus, lower levels of vitamin B12 and eGFR, and higher creatinine levels.

\section{Relationship between baseline homocysteine level and the incidence of short-term PSCI}

There was no significant difference in PSCI incidence at 3 months between unelevated and elevated associated with 3-month PSCI in females (OR 1.10, 95\% CI 0.61$1.98, P=0.75)$ and males (OR 1.22, 95\% CI 0.77-1.93, $P$ $=0.39$ ) (Table 3). The same pattern of results was observed when homocysteine was categorized as tertiles.

\section{Relationship between baseline homocysteine level and the incidence of long-term PSCI}

Compared to females with unelevated homocysteine, females with elevated homocysteine had a higher 12-month PSCI incidence rate (18.5\% vs. 40.0\%). There was no significant difference in 12 -month PSCI incidence between unelevated and elevated homocysteine in males (Figure 2). 
Li et al.: Homocysteine levels and post-stroke cognitive impairment

\begin{tabular}{|c|c|c|c|c|c|c|}
\hline \multirow[t]{2}{*}{ Baseline characteristics } & \multicolumn{2}{|l|}{ Overall } & \multicolumn{2}{|l|}{ Female } & \multicolumn{2}{|l|}{ Male } \\
\hline & $\begin{array}{l}\text { Female } \\
(n=156)\end{array}$ & $\begin{array}{l}\text { Male } \\
(n=428)\end{array}$ & $\begin{array}{l}\text { Hcy } \geq 15 \mu \mathrm{mol} / \mathrm{L} \\
(n=75)\end{array}$ & $\begin{array}{l}\text { Hcy }<15 \\
\mu \mathrm{mol} / \mathrm{L} \\
(n=81)\end{array}$ & $\begin{array}{l}\text { Hcy } \geq 15 \mu \mathrm{mol} / \mathrm{L} \\
(n=299)\end{array}$ & $\begin{array}{l}\text { Hcy }<15 \mu \mathrm{mol} / \mathrm{L} \\
(n=129)\end{array}$ \\
\hline Age (years), mean $\pm S D$ & $62.67 \pm 10.56$ & $60.38 \pm 11.56$ & $63.8 \pm 11.4$ & $61.6 \pm 9.7^{*}$ & $61.2 \pm 11.8$ & $58.5 \pm 10.8^{*}$ \\
\hline Education $\leq 9$ years $(\%)$ & $116(74.4)$ & $257(60.1)^{*}$ & $57(76.0)$ & $59(72.8)$ & $184(61.5)$ & $73(56.6)$ \\
\hline $\mathrm{BMI}\left(\mathrm{kg} / \mathrm{m}^{2}\right)$, mean $\pm \mathrm{SD}$ & $25.1 \pm 3.6$ & $24.9 \pm 3.2$ & $25.3 \pm 4.1$ & $24.9 \pm 3.0$ & $24.9 \pm 3.1$ & $25.0 \pm 3.5$ \\
\hline NIHSS, mean \pm SD & $1.6 \pm 1.4$ & $1.6 \pm 1.3$ & $1.5 \pm 1.2$ & $1.7 \pm 1.5$ & $1.5 \pm 1.2$ & $1.7 \pm 1.4$ \\
\hline \multicolumn{7}{|l|}{ Medical history } \\
\hline Diabetes mellitus (\%) & $41(26.3)$ & $79(18.5)^{*}$ & $21(28.0)$ & $20(24.7)$ & $38(12.7)$ & $41(31.8)^{*}$ \\
\hline Hypertension (\%) & $109(69.9)$ & $255(59.6)^{*}$ & $57(76.0)$ & $52(64.2)$ & $184(61.5)$ & $71(55.0)$ \\
\hline Hyperlipidemia (\%) & $18(11.5)$ & $52(12.2)$ & $7(9.3)$ & $11(13.6)$ & $31(10.4)$ & $21(16.3)$ \\
\hline History of stroke (\%) & $32(20.5)$ & $92(21.5)$ & $20(26.7)$ & $12(14.8)^{*}$ & $64(21.4)$ & $28(21.7)$ \\
\hline History of TIA (\%) & $10(6.4)$ & $26(6.07)$ & $4(5.3)$ & $6(7.4)$ & $18(6.0)$ & $8(6.2)$ \\
\hline History of CHD (\%) & $24(15.4)$ & $43(10.1)$ & $10(13.3)$ & $14(17.3)$ & $29(9.7)$ & $14(10.9)$ \\
\hline Current smoking (\%) & $4(2.6)$ & $204(47.7)^{*}$ & $3(4.0)$ & $1(1.2)$ & $144(48.2)$ & $60(46.5)$ \\
\hline Alcohol intake (\%) & $1(0.6)$ & $96(22.4)^{*}$ & $0(0.00)$ & $1(1.2)$ & $65(21.7)$ & $31(24.0)$ \\
\hline \multicolumn{7}{|l|}{ Laboratory results } \\
\hline Folate (nmol/L), median (IQR) & $8.4(4.3-15.0)$ & $6.3(3.4-13.0)^{*}$ & $8.8(4.5-14.6)$ & $7.9(3.6-17.0)$ & $6.3(3.3-12.1)$ & $6.5(3.4-15.6)$ \\
\hline $\begin{array}{l}\text { Vitamin B12 (pmol/L), } \\
\text { median (IQR) }\end{array}$ & $277(181-438)$ & $\begin{array}{l}228(161- \\
344)^{*}\end{array}$ & $243(157-393)$ & $\begin{array}{l}297(195- \\
493)^{*}\end{array}$ & $201(151-300)$ & $\begin{array}{l}292(210- \\
536)^{*}\end{array}$ \\
\hline Hcy $(\mu \mathrm{mol} / \mathrm{L})$, median (IOR) & $\begin{array}{l}14.2(11.6- \\
17.9)\end{array}$ & $\begin{array}{l}18.4(14.0- \\
25.0)^{*}\end{array}$ & $18(16.2-23.3)$ & $\begin{array}{l}11.8(10.2- \\
13.2)^{*}\end{array}$ & $\begin{array}{l}21.6(17.9- \\
29.0)\end{array}$ & $\begin{array}{l}12.7(11.3- \\
13.7)^{*}\end{array}$ \\
\hline $\begin{array}{l}\text { Creatinine }(\mu \mathrm{mol} / \mathrm{L}) \text {, median } \\
\text { (IOR) }\end{array}$ & $56(49-67)$ & $73(65-82)^{*}$ & $59(50-70)$ & $55(48.5-63)$ & $74(66-84)$ & $70.5(62-78)^{*}$ \\
\hline $\begin{array}{l}\text { eGFR }\left(\mathrm{mL} / \mathrm{min} / 1.73 \mathrm{~m}^{2}\right) \text {, } \\
\text { median (IOR) }\end{array}$ & $\begin{array}{l}93.5(82.7- \\
103.2)\end{array}$ & $\begin{array}{l}94.8(84.5- \\
103.3)\end{array}$ & $\begin{array}{l}89.7(77.7- \\
100.4)\end{array}$ & $\begin{array}{l}96.12(85.2- \\
103.7)\end{array}$ & $\begin{array}{l}92.96(82.4- \\
101.7)\end{array}$ & $\begin{array}{l}98.37(89.9- \\
106.6)^{*}\end{array}$ \\
\hline \multicolumn{7}{|l|}{ MTHFR C667T } \\
\hline CC (\%) & $30(19.6)$ & $95(23.2)$ & $10(13.9)$ & $20(24.7)$ & $64(22.1)$ & $31(25.8)^{*}$ \\
\hline CT (\%) & $67(43.8)$ & $186(45.4)$ & $30(41.7)$ & $37(45.7)$ & $113(39.0)$ & $73(60.8)$ \\
\hline TT (\%) & $56(36.6)$ & $129(31.5)$ & $32(44.4)$ & $24(29.6)$ & $113(39.0)$ & $16(13.3)$ \\
\hline $\begin{array}{l}\text { MoCA at } 3 \text { months, mean } \\
\pm S D\end{array}$ & $24.35 \pm 4.55$ & $25.02 \pm 4.39$ & $23.83 \pm 4.22$ & $24.83 \pm 4.82^{*}$ & $24.98 \pm 4.43$ & $25.1 \pm 4.31$ \\
\hline 3-month PSCI (\%) & $45(28.85)$ & $103(24.07)$ & $30(40.00)$ & $15(18.52)^{*}$ & $70(23.41)$ & $33(25.58)$ \\
\hline
\end{tabular}

${ }^{*}$ Significant difference between two groups, $P<0.05$.

BMI: body mass index; CHD: coronary heart disease; eGFR: estimated glomerular filtration rate; Hcy: homocysteine; IQR: interquartile range; MoCA: Montreal Cognitive Assessment; MTHFR: methylenetetrahydrofolate reductase; NIHSS: National Institutes of Health Stroke Scale; PSCI: post-stroke cognitive impairment; SD: standard deviation; TIA: transient ischemic attack.

Table 3 shows that female patients with elevated homocysteine had a higher risk of 12-month PSCI (OR 2.93, 95\% CI 1.42-6.07; $P=0.004)$, which was kept (OR $3.28,95 \%$ CI $1.47-7.34, P=0.004)$ when age, education, NIHSS, diabetes mellitus, smoking, baseline levels of folate, vitamin B12, eGFR, and MTHFR C667T genotype were adjusted. The findings based on homocysteine tertiles, elevated homocysteine levels monotonically increased the risk of PSCI $(P$ trend $=0.005)$. There was no relationship between homocysteine and the risk of 12-month PSCI in males. The same pattern of results was observed when homocysteine was categorized as tertiles.

The current findings remained the same when additional linear regression models were used to explore the relationships between baseline homocysteine and MoCA at 12-month follow-up. The linear regression results showed that higher homocysteine levels were associated with lower MoCA scores in females $(\beta=-0.54,95 \%$ CI -0.92 to $-0.16, P=0.006$; Table S3), but not in males $(\beta=0.32$, $95 \%$ CI $-0.08-0.72, P=0.113)$. In addition, a non-linear analysis also showed that the risk of developing PSCI increased with the rise of homocysteine concentration in females, but not in males (Figure S1).

\section{Relationship between homocysteine change at 3 months (homocysteine at 3 months minus baseline homocysteine) and the incidence of long- term PSCI}

The change in homocysteine at 3 months, named $\Delta \mathrm{Hcy}$, represented the homocysteine level at 3 months minus baseline homocysteine. A total of 391 patients with minor stroke and TIA were enrolled, consisting of 116 females and 275 males. In logistic regression, no significant 

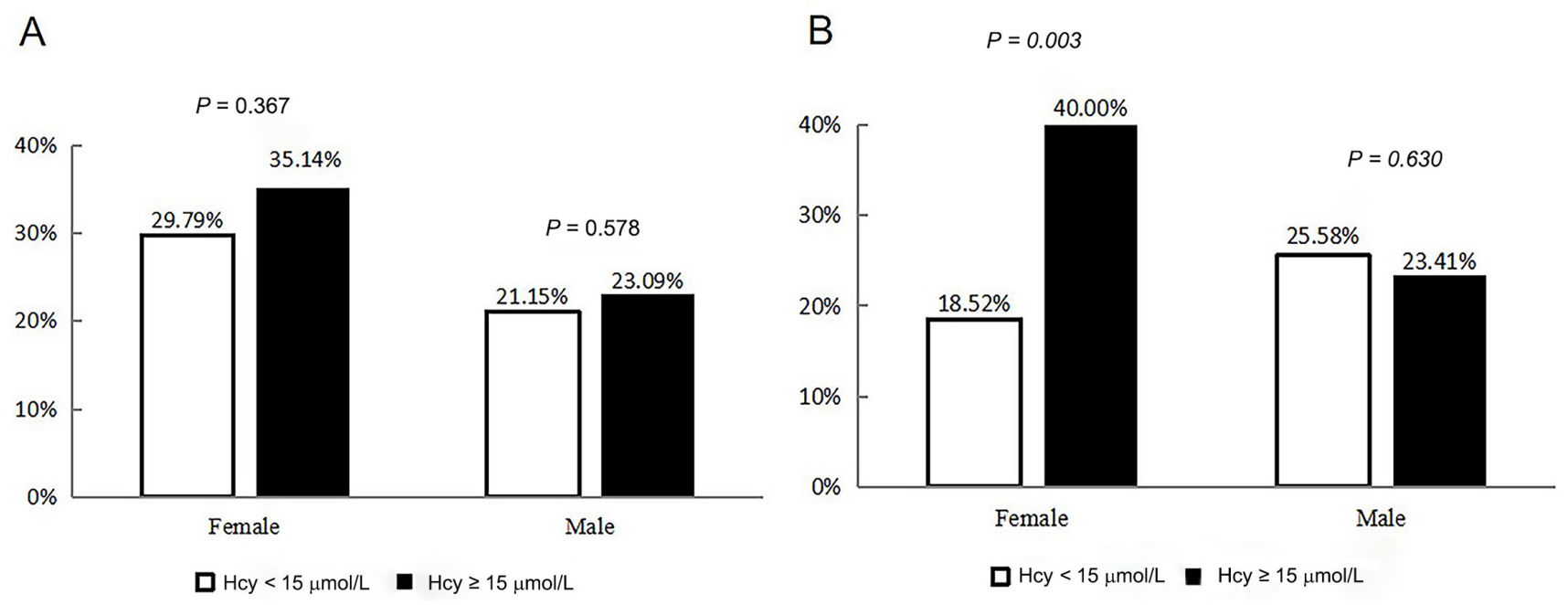

Figure 2. Incidence rates of post-stroke cognitive impairment at 3 months (A) and 12 months (B) grouped by sex and homocysteine levels. Hcy: homocysteine.

\begin{tabular}{|c|c|c|c|c|c|c|c|}
\hline & $\begin{array}{l}\text { Event } \\
n(\%)\end{array}$ & $\begin{array}{l}\text { Unadjusted } \\
\text { OR }(95 \% \mathrm{Cl})\end{array}$ & $P$-value & Model 1* (95\% Cl) & $P$-value & Model $2^{\dagger}(95 \% \mathrm{Cl})$ & $P$-value \\
\hline \multicolumn{8}{|l|}{ Female $(n=252)$} \\
\hline $\mathrm{Hcy}<15 \mu \mathrm{mol} / \mathrm{L}$ & $42(29.8)$ & Ref. & & Ref. & & Ref. & \\
\hline $\mathrm{Hcy} \geq 15 \mu \mathrm{mol} / \mathrm{L}$ & $39(35.1)$ & $1.28(0.75-2.17)$ & 0.37 & $1.3(0.7-2.4)$ & 0.46 & $1.10(0.61-1.98)$ & 0.75 \\
\hline \multicolumn{8}{|l|}{ Tertile of Hcy } \\
\hline Lowest tertile & $22(26.8)$ & Ref. & & Ref. & & Ref. & \\
\hline Intermediate tertile & 31 (36.9) & $1.60(0.83-3.09)$ & 0.17 & $1.67(0.8-3.6)$ & 0.20 & $1.38(0.68-2.82)$ & 0.38 \\
\hline Highest tertile & $28(32.6)$ & $1.32(0.68-2.56)$ & 0.42 & $1.30(0.6-3.0)$ & 0.54 & $1.13(0.54-2.37)$ & 0.75 \\
\hline \multicolumn{8}{|l|}{ Male $(n=667)$} \\
\hline $\mathrm{Hcy}<15 \mu \mathrm{mol} / \mathrm{L}$ & $44(21.2)$ & Ref. & & Ref. & & Ref. & \\
\hline $\mathrm{Hcy} \geq 15 \mu \mathrm{mol} / \mathrm{L}$ & $106(23.1)$ & $1.12(0.75-1.67)$ & 0.58 & $1.41(0.86-2.31)$ & 0.17 & $1.22(0.77-1.93)$ & 0.39 \\
\hline \multicolumn{8}{|l|}{ Hcy tertiles } \\
\hline Lowest tertile & $50(22.6)$ & Ref. & & Ref. & & Ref. & \\
\hline Intermediate tertile & $48(21.8)$ & $0.95(0.61-1.50)$ & 0.84 & $1.12(0.66-1.90)$ & 0.69 & $0.94(0.58-1.54)$ & 0.81 \\
\hline Highest tertile & $52(23.0)$ & $1.02(0.66-1.59)$ & 0.92 & $1.38(0.78-2.43)$ & 0.27 & $1.26(0.75-2.13)$ & 0.38 \\
\hline
\end{tabular}

${ }^{*}$ Adjusted for age, education, NIHSS, diabetes mellitus, current smoking, baseline levels of folate, vitamin B12, eGFR, and MTHFR genotype. ${ }^{\dagger}$ After imputation and adjusted the same factors with Model 1. Cl: confidence interval; eGFR: estimated glomerular filtration rate; Hcy: homocysteine; MTHFR: methylenetetrahydrofolate reductase; NIHSS: National Institutes of Health Stroke Scale; OR: odds ratio; PSCl: post-stroke cognitive impairment.

relationship was found between $\Delta \mathrm{Hcy}$ and the risk of PSCI at 12 months in females and males using the crude model and the adjusted model (Table S4).

\section{DISCUSSION}

In this study, we demonstrated that females had lower baseline homocysteine levels than males. Elevated homocysteine levels at baseline had no relationship with PSCI at short term (3 months). Elevated homocysteine levels were significantly associated with the risk of long-term (12-month) PSCI in females with minor acute ischemic stroke or TIA, but not in males. Additional adjustment for sociodemographics and vascular risk factors still supported the relationship between homocysteine and PSCI. Linear regression analysis and restricted cubic spline (non-linear) analysis further confirmed the sex-specific relationship between homocysteine and 12-month follow-up PSCI.

In the current study, we conducted cognitive follow-ups at two time points ( 3 and 12 months), which allowed us to explore both short- and long-term effects of homocysteine on the PSCI. Different factors were associated with PSCI in the short term and long term. ${ }^{[23,24]}$ Previous observational studies using a small sample size $(<200 \text { subjects })^{[10,11]}$ demonstrated that homocysteine was associated with vascular dementia or PSCI in the short term. However, a prospective cohort study ${ }^{[9]}$ or a case-control study ${ }^{[12]}$ based on large sample sizes (200-300 cases) demonstrated that 
Li et al.: Homocysteine levels and post-stroke cognitive impairment

\begin{tabular}{|c|c|c|c|c|c|c|c|}
\hline & $\begin{array}{l}\text { Event } \\
n(\%)\end{array}$ & $\begin{array}{l}\text { Unadjusted } \\
\text { OR }(95 \% \mathrm{CI})\end{array}$ & $P$-value & Model 1* $(95 \% \mathrm{Cl})$ & $P$-value & Model $2^{\dagger}(95 \% \mathrm{Cl})$ & $P$-value \\
\hline \multicolumn{8}{|l|}{ Female $(n=156)$} \\
\hline $\mathrm{Hcy}<15 \mu \mathrm{mol} / \mathrm{L}$ & $15(18.5)$ & Ref. & & Ref. & & Ref. & \\
\hline $\mathrm{Hcy} \geq 15 \mu \mathrm{mol} / \mathrm{L}$ & $30(40.0)$ & $2.93(1.42-6.07)$ & 0.004 & $5.06(2.01-12.74)$ & 0.001 & $3.28(1.47-7.34)$ & 0.004 \\
\hline \multicolumn{8}{|l|}{ Tertile of Hcy } \\
\hline Lowest tertile & $5(10.2)$ & Ref. & & Ref. & & Ref. & \\
\hline Intermediate tertile & $21(38.9)$ & $5.60(1.91-16.40)$ & 0.002 & $15.01(3.43-65.77)$ & $<0.001$ & $8.33(2.49-27.87)$ & 0.001 \\
\hline Highest tertile & 19 (35.9) & $4.92(1.67-14.51)$ & 0.004 & $17.94(3.72-85.56)$ & $<0.001$ & $7.76(2.24-26.94)$ & 0.001 \\
\hline \multicolumn{8}{|l|}{ Male $(n=428)$} \\
\hline $\mathrm{Hcy}<15 \mu \mathrm{mol} / \mathrm{L}$ & $33(25.6)$ & Ref. & & Ref. & & Ref. & \\
\hline $\mathrm{Hcy} \geq 15 \mu \mathrm{mol} / \mathrm{L}$ & $70(23.4)$ & $0.89(0.55-1.43)$ & 0.63 & $1.10(0.59-2.02)$ & 0.78 & $0.86(0.49-1.49)$ & 0.59 \\
\hline \multicolumn{8}{|l|}{ Hcy tertiles } \\
\hline Lowest tertile & $37(26.2)$ & Ref. & & Ref. & & Ref. & \\
\hline Intermediate tertile & $39(27.1)$ & $1.04(0.62-1.77)$ & 0.87 & $1.16(0.62-2.19)$ & 0.66 & $0.89(0.50-1.59)$ & 0.70 \\
\hline Highest tertile & 27 (18.9) & $0.65(0.37-1.15)$ & 0.14 & $0.74(0.35-1.56)$ & 0.43 & $0.69(0.35-1.35)$ & 0.27 \\
\hline
\end{tabular}

*Adjusted for age, education, NIHSS, diabetes mellitus, current smoking, baseline levels of folate, vitamin B12, eGFR, and MTHFR genotype. ${ }^{\dagger}$ After imputation and adjusted the same factors with Model 1. Cl: confidence interval; eGFR: estimated glomerular filtration rate; Hcy: homocysteine; MTHFR: methylenetetrahydrofolate reductase; NIHSS: National Institutes of Health Stroke Scale; OR: odds ratio; PSCl: post-stroke cognitive impairment.

there was no association between homocysteine and PSCI at 3-6 months, which is consistent with the findings in our study using data from a large national prospective stroke cohort (943 cases).

We found that elevated homocysteine was associated with long-term (12-month) PSCI, but not with short-term (3-month) PSCI in females. There are two consensuses that put forward the concept of classifying PSCI according to the time of appearance of cognitive impairment after stroke. ${ }^{[25,26]}$ It is divided into early-onset (3-6 months after stroke) and delayed-onset (6 months or more after stroke) PSCI, which have different mechanisms. Early-onset PSCI depends on a complex interplay between features of the stroke lesion, such as large size or strategic site, and brain resilience, such as cognitive reserve and brain reserve. Delayed-onset PSCI is driven mostly by severe small vessel disease or concurrent Alzheimer disease pathology. Neuroimaging studies have demonstrated that homocysteine contributes to small vessel disease, including white matter lesions, silent infarcts, and brain atrophy. ${ }^{[27,28]}$ Also, homocysteine showed significant associations with Alzheimer's disease and homocysteine-lowering treatments are recommended to prevent Alzheimer disease in randomized controlled trials and meta-analysis. ${ }^{[5,29]}$ So we speculated that elevated homocysteine might promote PSCI through small vessel disease or Alzheimer disease pathology, which may explain our finding that homocysteine levels are associated with long-term incidence of PSCI.
In the current study, elevated homocysteine was associated with PSCI in females, but not in males. Accumulating evidence suggests that homocysteine promoted white matter lesions and PSCI pathogenesis by endothelial dysfunction. ${ }^{[30-32]}$ Estrogen is thought to protect vascular endothelial cells. ${ }^{[33]}$ In postmenopausal females, the estrogen levels decrease ${ }^{[13]}$ and homocysteine levels increase, ${ }^{[33]}$ which may aggravate endothelial dysfunction and speed up the progress of PSCI. In the present study, average age of females was above 60 years, which is a postmenopausal state. The decreasing estrogen levels in females, which leads to elevated homocysteine levels and weakened endothelial protective effect, may be one explanation for the sex difference observed in the association of homocysteine and PSCI. Elevated homocysteine caused by low folic acid was reported to have a higher risk of the 5-year composite vascular outcome, including vascular dementia, vascular cognitive impairment, or fatal stroke among females, but not in males, ${ }^{[34]}$ which could support the findings in our study. Other evidences showed that females were more susceptible to the damaging effect of homocysteine in PSCI than males, which could also be supported by previous clinical findings that elevated homocysteine was a stronger risk factor in females than in males in vascular disease, ${ }^{[33]}$ including ischemic stroke incidence, ${ }^{[15]}$ poor prognosis (death and major disability) after stroke onset, ${ }^{[16]}$ and neurodegenerative cognitive impairment. ${ }^{[17]}$ 
Our research had several limitations. Firstly, although folate, vitamin B12, renal function, and MTHFR genotype were taken into consideration, homocysteine levels are also affected by diet, vitamin supplements, gastrointestinal factors that lead to B vitamins' malabsorption, and other genetic mutations (e.g., the cystathionine $\beta$-synthase genotype), of which data were not collected during the follow-up. Secondly, magnetic resonance imaging is an efficient instrument to evaluate brain lesions. ${ }^{[3]}$ However, brain magnetic resonance imaging data of 12 -month follow-up were not available. Therefore, we cannot evaluate brain structure changes, especially white matter lesions and brain atrophy, which might be associated with high homocysteine levels and cognitive impairments. Further studies are warranted to evaluate the potential intermediary role of brain structural changes in the relationship between homocysteine and long-term PSCI. Thirdly, one of the inclusion criteria was to be enrolled within 7 days after the onset of stroke or TIA in this prospective registry ICONS study. The mean (interquartile range) hours from event onset to hospitalization was 13.6 (3.2-46.7) in patients with 3-month cognitive follow-up and 16.4 (3.3-47.0) with 12-month cognitive follow-up. The blood samples used to test homocysteine were obtained on the second day of hospitalization. Hence, there was time delay from stroke or TIA onset to admission homocysteine levels. Although due to the practical limitation of the time interval between hospitalizations of stroke patients, homocysteine in similar studies also had a certain time delay. ${ }^{[7,22,36]}$ This may be a factor to accurately reflect the levels of homocysteine at stroke onset. Collecting blood samples within 24 hours after the stroke onset in further studies may remedy this limitation. Fourthly, 45.9\% (491/1070) of patients were lost to follow-up in MoCA and could not be included in the long-term PSCI analysis. Compared with the included population, the excluded population has milder stroke symptoms, lower homocysteine levels, and higher folic acid and vitamin B12 levels, which demonstrated that there is a selection bias due to loss to follow-up. The median homocysteine of the included group with 12-month MoCA was $17.2 \mu \mathrm{mol} / \mathrm{L}$. The research conclusions based on the included group are restrictive to the population with high homocysteine and cannot be extended to other populations.

In conclusion, elevated homocysteine levels at baseline were independently associated with 12-month PSCI in female patients with minor acute ischemic stroke or TIA, which should be further validated in future clinical studies.

\section{Source of Funding}

Ethics approval and consent to participate
The protocol of the ICONS study was approved by the ethics committee of a hospital (blinded as requested; Institutional Review Board approval number KY2015001-01) and all participating centers. All participants obtained written informed consent from patients or legally authorized representatives.

This work was supported by the grants from National Key R\&D Program of China (2016YFC0901002, 2018YFC1312903), National Science and Technology Major Project (2017ZX09304018), Beijing Municipal Science \& Technology Commission (D171100003017002, Z181100001818001), National Natural Science Foundation of China (81870821, 81471215, 82071187), and Beijing Youth Talent Team Support Program (2018000021223TD08).

\section{Acknowledgments}

We thank the staff and participants of the CSCA and CNSR-III studies for their contribution. We thank Yueran Li (University of California, Berkeley, CA, USA) for language editing.

\section{Conflicts of interest}

None

\section{REFERENCES}

1. Pendlebury ST, Rothwell PM. Incidence and prevalence of dementia associated with transient ischaemic attack and stroke: Analysis of the population-based oxford vascular study. Lancet Neurol 2019;18:248-58

2. Turner GM, McMullan C, Atkins L, Foy R, Mant J, Calvert M. Tia and minor stroke: A qualitative study of long-term impact and experiences of follow-up care. BMC Fam Pract 2019;20:176.

3. van Rooij FG, Kessels RP, Richard E, De Leeuw FE, van Dijk EJ. Cognitive impairment in transient ischemic attack patients: A systematic review. Cerebrovasc Dis 2016;42:1-9

4. Miller JW. Homocysteine - what is it good for?. J Intern Med 2021;290:934-6.

5. Yu JT, Xu W, Tan CC, Andrieu S, Suckling J, Evangelou E, et al. Evidencebased prevention of Alzheimer's disease: Systematic review and metaanalysis of 243 observational prospective studies and 153 randomised controlled trials. J Neurol Neurosurg Psychiatry 2020;91:1201-9.

6. David Spence J. Advances in stroke prevention. J Transl Int Med 2018;6:105-14.

7. Li J, Jiang S, Zhang Y, Tang G, Wang Y, Mao G, et al. H-type hypertension and risk of stroke in chinese adults: A prospective, nested case-control study. J Transl Int Med 2015;3:171-8.

8. Hankey G. Is homocysteine a causal and treatable risk factor for vascular diseases of the brain (cognitive impairment and stroke)? Ann Neurol 2002;51:279-81.

9. Barba R, Martinez-Espinosa S, Rodriguez-Garcia E, Pondal M, Vivancos J, Del Ser T. Poststroke dementia : Clinical features and risk factors. Stroke 2000;31:1494-501.

10. Jiang B, Chen Y, Yao G, Yao C, Zhao H, Jia X, et al. Effects of differences in serum total homocysteine, folate, and vitamin B12 on cognitive 
impairment in stroke patients. BMC Neurol 2014;14:217.

11. Khedr E, Hamed S, El-Shereef H, Shawky O, Mohamed K, Awad E, et al. Cognitive impairment after cerebrovascular stroke: Relationship to vascular risk factors. Neuropsychiatr Dis Treat 2009;5:103-16.

12. Sachdev P, Brodaty H, Valenzuela M, Lorentz L, Looi J, Berman K, et al. Clinical determinants of dementia and mild cognitive impairment following ischaemic stroke: The sydney stroke study. Dement Geriatr Cogn Disord 2006;21:275-83.

13. Gaikwad N. Mass spectrometry evidence for formation of estrogenhomocysteine conjugates: Estrogens can regulate homocysteine levels. Free Radic Biol Med 2013;65:1447-54.

14. Lobo R. Homocysteine in women's health. Menopause. 2003;10:271-3.

15. Wang C, Han L, Wu Q, Zhuo R, Liu K, Zhao J, et al. Association between homocysteine and incidence of ischemic stroke in subjects with essential hypertension: A matched case-control study. Clin Exp Hypertens 2015;37:557-62.

16. Zhong C, Xu T, Xu T, Peng Y, Wang A, Wang J, et al. Plasma homocysteine and prognosis of acute ischemic stroke: A gender-specific analysis from catis randomized clinical trial. Mol Neurobiol 2017;54:2022-30.

17. Bakeberg M, Jefferson A, Riley M, Byrnes M, Ghosh S, Mastaglia F, et al. Elevated serum homocysteine levels have differential gender-specific associations with motor and cognitive states in parkinson's disease. Parkinsons Dis 2019;2019:3124295.

18. Rong D, Liu J, JiaX, Al-Nafisee D, JiaS, Sun G, et al. Hyperhomocysteinaemia is an independent risk factor for peripheral arterial disease in a chinese han population. Atherosclerosis 2017;263:205-10.

19. Wang $Y$, Liao X, Wang C, et al. Impairment of cognition and sleep after acute ischaemic stroke or transient ischaemic attack in Chinese patients: design, rationale and baseline patient characteristics of a nationwide multicentre prospective registry. Stroke Vasc Neurol 2021;6:139-44.

20. Fischer U, Baumgartner A, Arnold M, Nedeltchev K, Gralla J, De Marchis G, et al. What is a minor stroke? Stroke 2010;41:661-6.

21. Cotton F, Wautrecht J, Léchevin V, Macours P, Thiry P, Gervy C, et al. Reference intervals for plasma homocysteine by the axsym immunoassay after collection in fluoride tubes. Clin Chem 2003;49:315-7.

22. Zhao M, Wang X, He M, Qin X, Tang G, Huo Y, et al. Homocysteine and stroke risk: Modifying effect of methylenetetrahydrofolate reductase c677t polymorphism and folic acid intervention. Stroke 2017;48:1183-90.

23. Huang Y, Yang S, Jia J. Factors related to long-term post-stroke cognitive impairment in young adult ischemic stroke. Med Sci Monit 2015;21:65460.

24. Ballard C, Rowan E, Stephens S, Kalaria R, Kenny RA. Prospective follow-up study between 3 and 15 months after stroke: Improvements and decline in cognitive function among dementia-free stroke survivors $>75$ years of age. Stroke 2003;34:2440-4.

25. Chinese Stroke Association Post-stroke Cognitive Impairment Research Round Table Expert Group. [Experts consensus on research on prevention and treatment of poststroke cognitive impairment in China]. Zhongguo Zuzhong Zazhi 2020;15:158-66.

26. Mok VC, Lam BY, Wong A, Ko H, Markus HS, Wong LK. Early-onset and delayed-onset poststroke dementia - revisiting the mechanisms. Nat Rev Neurol 2017;13:148-59.

27. Firbank M, Narayan S, Saxby B, Ford G, O'Brien JJIp. Homocysteine is associated with hippocampal and white matter atrophy in older subjects with mild hypertension. Int Psychogeriatr 2010;22:804-11.

28. Wright C, Paik M, Brown T, Stabler S, Allen R, Sacco R, et al. Total homocysteine is associated with white matter hyperintensity volume: The northern manhattan study. Stroke 2005;36:1207-11.

29. Seshadri S. Elevated plasma homocysteine levels: Risk factor or risk marker for the development of dementia and Alzheimer's disease? J Alzheimers Dis 2006;9:393-8.

30. Jakubowski H. Homocysteine modification in protein structure/function and human disease. Physiol Rev 2019;99:555-604.

31. Hankey G. Is homocysteine a causal and treatable risk factor for stroke? Lancet Neuro 2007;6:751-2.

32. Moretti R, Caruso P. The Controversial Role of Homocysteine in Neurology: From Labs to Clinical Practice. Int J Mol Sci 2019;20:231.

33. Verhoef P. Hyperhomocysteinemia and risk of vascular disease in women. Semin Thromb Hemost 2000;26:325-34.

34. Maxwell C, Hogan D, Ebly EJD, disorders gc. Serum folate levels and subsequent adverse cerebrovascular outcomes in elderly persons. Dement Geriatr Cogn Disord 2002;13:225-34.

35. Sanoussi S, Comet C, Kaefer K, Attou R, De Bels D, Gazagnes MD, et al. Can magnetic resonance imaging make the differential diagnosis between cerebral ischemia and epilepsy? J Transl Int Med 2019;7:123-5.

36. Li J, Wang Y, Li H, Zuo Z, Lin J, Wang A, et al. Homocysteine level predicts response to dual antiplatelet in women with minor stroke or transient ischemic attack: subanalysis of the chance trial. Arterioscler Thromb Vasc Biol 2020;40:839-46.

How to cite this article: Li R, Weng $\mathrm{H}$, Pan $\mathrm{Y}$, Meng $\mathrm{X}$, Liao X, Wang $M$, et al. Relationship between homocysteine levels and post-stroke cognitive impairment in female and male population: from a prospective multicenter study. J TransI Intern Med 2021; 9: 264-72. 